\title{
CDKN2A NP_000068.1:p.V51I
}

National Cancer Institute

\section{Source}

National Cancer Institute. CDKN2A NP 000068.1:p.V511. NCI Thesaurus. Code C146932.

A change in the amino acid residue at position 51 in the cyclin-dependent kinase inhibitor

2 protein where valine has been replaced by isoleucine. 